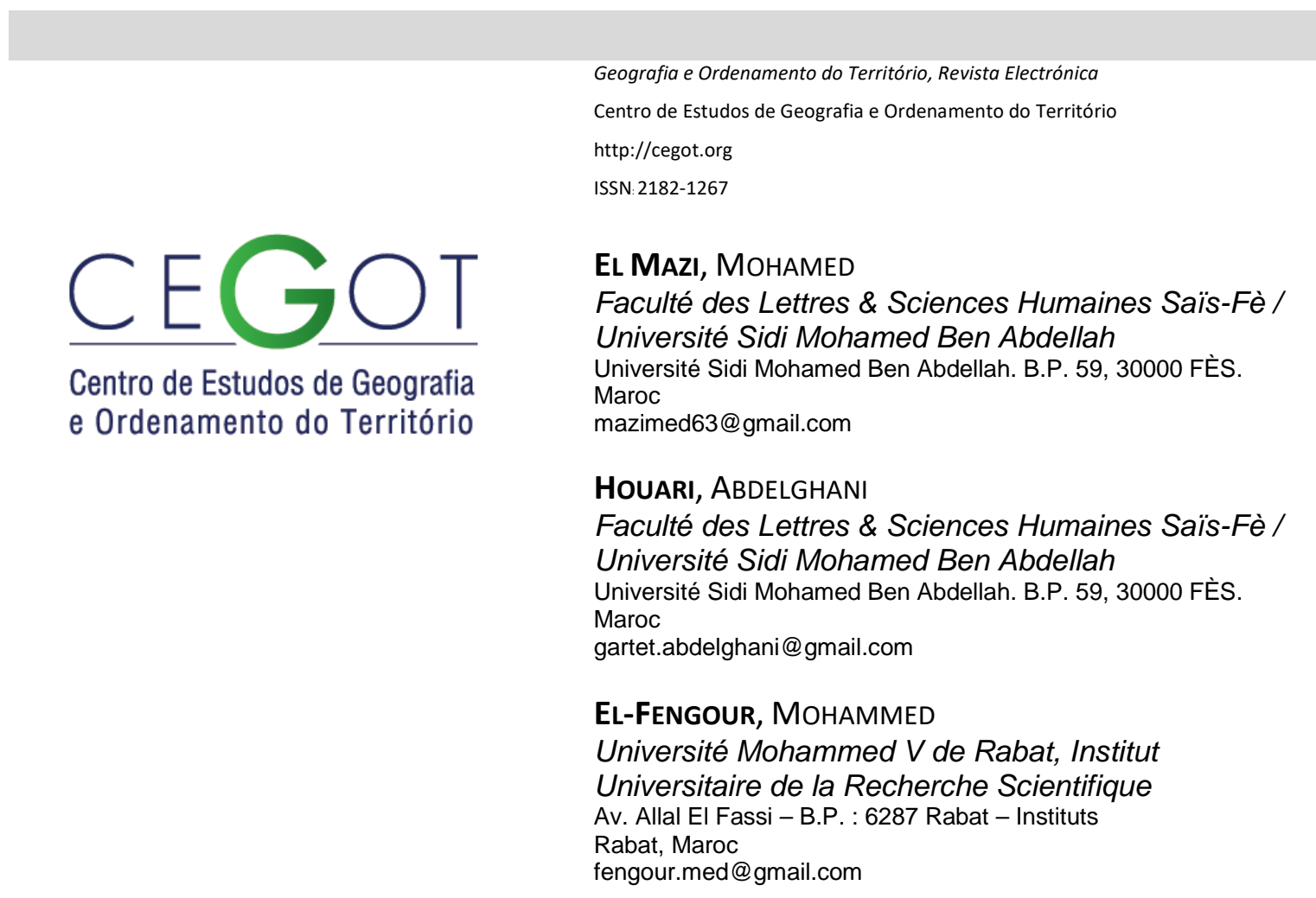

\title{
Apport de la télédétection et du SIG au suivi de la dynamique spatiotemporelle des forêts dans le massif numidien de Jbel Outka (Rif central, Maroc)
}

\author{
Contribuição do sensoriamento remoto e dos SIG para monitorizar as \\ dinâmicas temporais e espaciais da floresta de Jebel Outka montanha (Central \\ Rif, Marrocos) \\ Contribution of remote sensing and GIS to monitor the spatiotemporal \\ dynamics of Jebel Outka Mountain forest (Central Rif, Morocco)
}

Referência: El Mazi, Mohamed; Houari, Abdelghani; El-Fengour, Mohammed (2017). Apport de la télédétection et du SIG au suivi de la dynamique spatiotemporelle des forêts dans le massif numidien de Jbel Outka (Rif central, Maroc). Revista de Geografia e Ordenamento do Território (GOT), n.o 11 (junho). Centro de Estudos de Geografia e Ordenamento do Território, p. 171-187, dx.doi.org/10.17127/got/2017.11.008

\section{RESUMÉ}

Les écosystèmes forestiers du massif numidien de Jbel Outka situé au cœur du Rif Central au nord du Maroc, ont connu, au cours des dernières décennies, de grandes pressions aussi bien naturelles qu'anthropiques conduisant à des changements observables dans le paysage forestier. Néanmoins la nature et l'intensité de ces changements restent peu connues. L'objectif de cette étude est d'évaluer la dynamique du paysage forestier du massif numidien de Jbel d'Outka, et d'en expliquer les causes, en se basant sur une approche 
diachronique repose sur l'intégration et traitement des données multidates et multisources, à l'aide du Système d'Information Géographique (SIG), et des logiciels de la Télédétection spatiale. Les résultats obtenus soulignent une forte régression dans les dynamiques de la forêt.

Mots clé : Photographie aérienne; images satellitaires; télédétection; SIG ; milieu forestier ; Jbel Oudka ; Rif Central.

\section{RESUMO}

Os ecossistemas do maciço florestal númido da montanha Outka localizam-se no Rif Central no norte de Marrocos e têm, nas últimas décadas, altas pressões naturais e antropogénicas, levando a alterações observáveis na paisagem florestal. A natureza e a intensidade destas mudanças permanecem pouco conhecidas. O objetivo deste estudo é avaliar a dinâmica da paisagem florestal do maciço Outka da montanha Outka e explicar as suas causas, com base numa abordagem diacrónica, usando a integração e processamento de dados multidate e multi-fonte, usando GPS e software de sensoriamento remoto do espaço. Os resultados obtidos mostram uma forte regressão na dinâmica da floresta.

Palavras-chave: Fotografia aérea; Imagens de satélite; Sensoriamento remoto; SIG, floresta de montanha Outka; Montanha de Rif central

\section{ABSTRACT}

The forest ecosystems of the Numidian massif of Outka mountain are located in the Central Rif in northern Morocco, and have got, over the past decades, high natural and anthropogenic pressures, leading to observable changes in the forest landscape. The nature and the intensity of these changes remain little known. The objective of this study is to assess the dynamics of the forest landscape of the Outka massif of Outka mountain, and explain their causes, based on a diachronic approach, using the integration and processing of multidate and multisource data, Using GPS and Space Remote Sensing software. The results obtained show a strong regression in the dynamics of the forest.

Keywords: Aerial photography; Satellite imagery; Remote Sensing; GIS, Outka mountain forest; Central Rif Mountain.

\section{Introduction}

Les peuplements forestiers, préforestiers et prés steppiques du Maroc subissent depuis fort longtemps une pression anthropique plus ou moins importante selon les régions. L'équilibre naturel a été rompu pour un grand nombre de milieux. C'est le cas en particulier de ceux qui sont écologiquement fragiles du fait qu'ils se situent dans des conditions écologiques marginales (Benabid, A. 1984). 
Dans le cas des montagnes de Rif central, la dégradation des peuplements forestiers est aujourd'hui de plus en plus alarmante. Ce constat est dû à des facteurs naturels notamment climatiques, en corrélation avec la pression anthropique exercée sur les écosystèmes forestiers. L'action anthropique se manifeste par les coupes de bois, le surpâturage, l'incendie et le défrichement. Cette pression est aggravée notamment depuis les années 80 du siècle dernier à cause de l'accroissement démographique rapide et le recours aux cultures illicites du kif (Cannabis), qui représentent près de $90 \%$ du processus de déforestation dans les montagnes rifaines.

Ce travail prétend servir à quantifier la dégradation des milieux forestiers dans le Rif central à partir de l'étude d'une zone pilote; il s'agit de la forêt de Jbel Outka située dans le Rif central. La méthodologie s'appuie sur une analyse diachronique basée sur la comparaison de documents anciens (photographies aériennes de 1962) avec des documents récents (image satellitaire Landsat de $30 \mathrm{~m}$ ), sur le travail de terrain et en se basant sur les outils de la recherche géographique notamment les Systèmes d'information géographique (SIG) et la télédétection spatiale. Les résultats de cette étude prétendent de préciser les causes de cette dynamique et ses impacts sur les milieux naturels.

\section{Présentation de la zone d'étude}

Le Jbel Outka est un massif numidien dans le Rif central, il fait partie du bassin versant de l'Ouerrha. Cette situation lui confère une extrême diversité sur le plan topographique, avec des altitudes allant de 200 à 1600 mètres. Elle est délimitée au Nord par l'Oued Maachoq, au Sud par Ghafsai, à l'Est par l'Oued Amzaz et à l'Ouest par l'Oued Aoulai. Sur le plan forestier, ladite forêt est gérée par le biais des services provinciaux respectifs des eaux et Forêts de Taounate et d'Al Hoceima qui relève de la Direction régionale des eaux et forêts de Taza. La gestion directe est assurée par les triages de Outka I, Outka II, Talensift et Arguiouènne. 


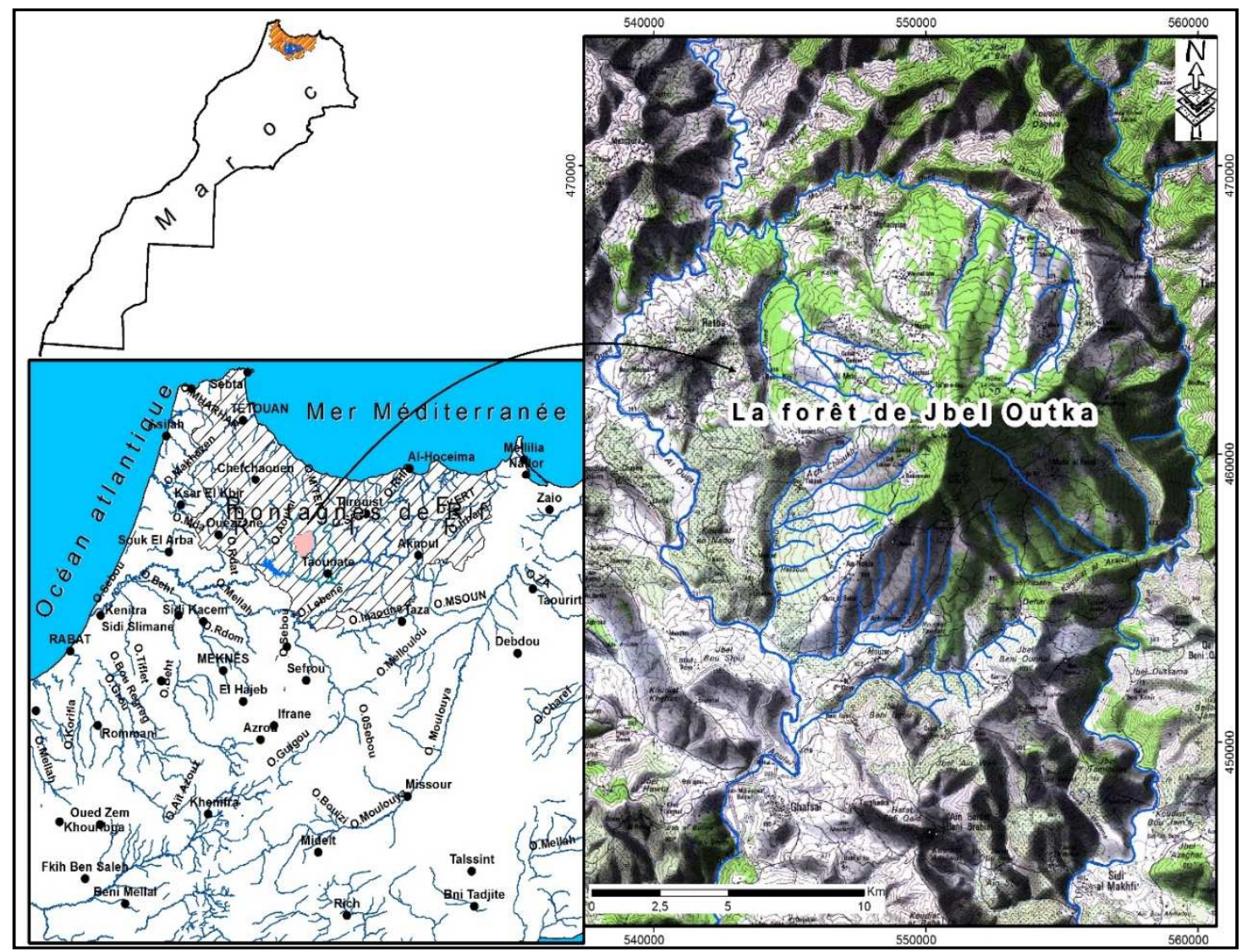

Figure 1 - Situation géographique de la forêt de Jbel Outka

Grâce à sa position géographique privilégiée, le Jbel Outka est parmi les massifs les plus arrosés au Maroc, avec une pluviométrie annuelle moyenne dépassant les 1400 mm/an. Cette moyenne fluctue entre $753 \mathrm{~mm}$ comme valeur minimale enregistrée en l'an 2000 et 3117 mm observée en 1997. L'effet de l'altitude est très marqué et la variabilité spatiale des précipitations est nettement perceptible. Les températures ont une moyenne minimale de $3^{\circ} \mathrm{C}$ en saisons froides et une moyenne maximale de $36^{\circ} \mathrm{Cen}$ saisons chaudes.

Suivant la classification synthétique d'Emberger, la forêt du Jbel Outka se caractérise par un gradient bioclimatique allant du subhumide à perhumide, le subhumide à hiver tempéré et chaud. Au niveau de cette ambiance, où l'on trouve des peuplements de chêne vert, le subhumide à hiver froid a tempéré correspond à l'ère de répartition de la subéraie et dans le bioclimat humide à perhumide avec variante froide a tempéré, on retrouve les peuplements de chêne Tauzin et chêne Zéen.

Du point de vue des étages de végétation, on relève la représentation des étages: thermoméditerraéen à l'ouest de la forêt, mésoméditerranéen qui englobe l'aire de la 
subéraie avec présence de l'arbousier et l'étage supraméditerranéen caractérisé par l'extension du chêne Tauzin.

La situation climatique favorable au développement les formations forestières est accompagnée par une variation des faciès lithologiques. Ceux-ci sont composés essentiellement par des flyschs à banc de grès siliceux correspondant au Crétacé inférieur, des grès siliceux grossiers et épais Numidien et par un mélange de Flysch marneux du crétacé supérieur et de formations superficielles du Quaternaire observés sous forme de dépôts au niveau des pentes fortes et des hauts niveaux érodés.

De point de vue pédologique, les sols rencontrés au niveau de Jbel Outka sont à base d'unités simples constituées de sols peu évolués ou sols brunifiés et d'unités complexes à base de sol peu évolués et minéraux bruts ou sols peu évolués brunifiés ou calcimagnésiques.

Il faut également noter que la situation géographique, l'abondance des précipitations, l'opposition topographique et des faciès lithologiques à tendance de grès numidien, favorisent une richesse forestière luxuriante (Benabid,1984). Surtout pour le chêne zéen et le chêne tauzin qui constitue à Jbel Outka $34 \%$ du total de cette espèce au niveau national (Alouane, 1994). Le chêne liège est plus étendu que la précédente et atteint des altitudes qui peuvent dépasser les $900 \mathrm{~m}$. Le chêne vert associe selon les conditions écologiques d'autres essences dites secondaires tels lentisque, Phyillyrea media, Arbustes unedo.

\section{Matériels et approche méthodologique}

\subsection{Présentation des données}

Les documents utilisés dans cette étude sont :

- Les photographies aériennes panchromatiques d'échelle 1/20 000 des missions d'août 1962 et septembre 2006, avec une résolution spatiale de $1(\mathrm{~m})$;

- Les images Landsat de juillet 2012 avec une résolution spatiale de $30 \mathrm{~m}$;

- Un modèle numérique de terrain MNT, avec une résolution spatiale de $30 \mathrm{~m}$;

- Les cartes topographiques de Ghafsai et de Ketama au 1/100000 de 1994. 


\subsection{Approche Méthodologique}

La méthodologie adoptée dans cette étude repose sur 4 étapes principales, résumés dans l'organigramme méthodologique suivant (Fig. 2).

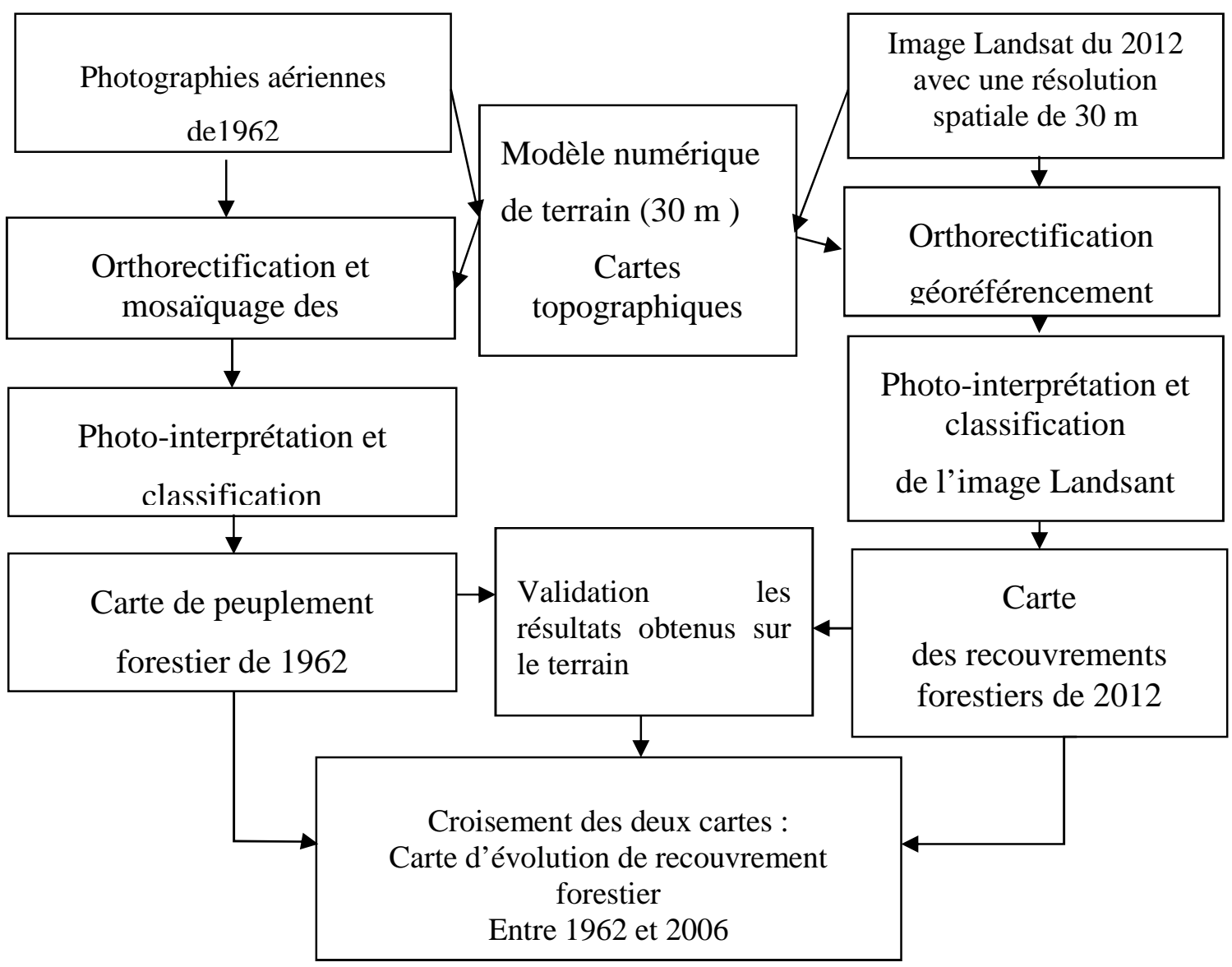

Figure 2- Organigramme méthodologique

\subsubsection{Prétraitement}

La photographie aérienne désigne toute vue prise depuis un engin se trouvant dans l'atmosphère. C'est un document brut qui subit l'ensemble des déformations, dues aux variations du relief, à la caméra, à la sphéricité de la terre, aux déformations radiales, etc (Labhar, 2011). Pour corriger ces distorsions les images ont subi une orthorectification à l'aide des logiciels de traitement d'image, en se basant sur l'image de référence (les cartes topographiques, les images satellitaire, MNT), afin d'extraire les cordonnées $X, Y$ et $Z$ de 
chaque point de contrôle, ensuite ces photos ont été assemblées en mosaïques de manière à recouvrir toute la zone d'étude (Fig. 3).

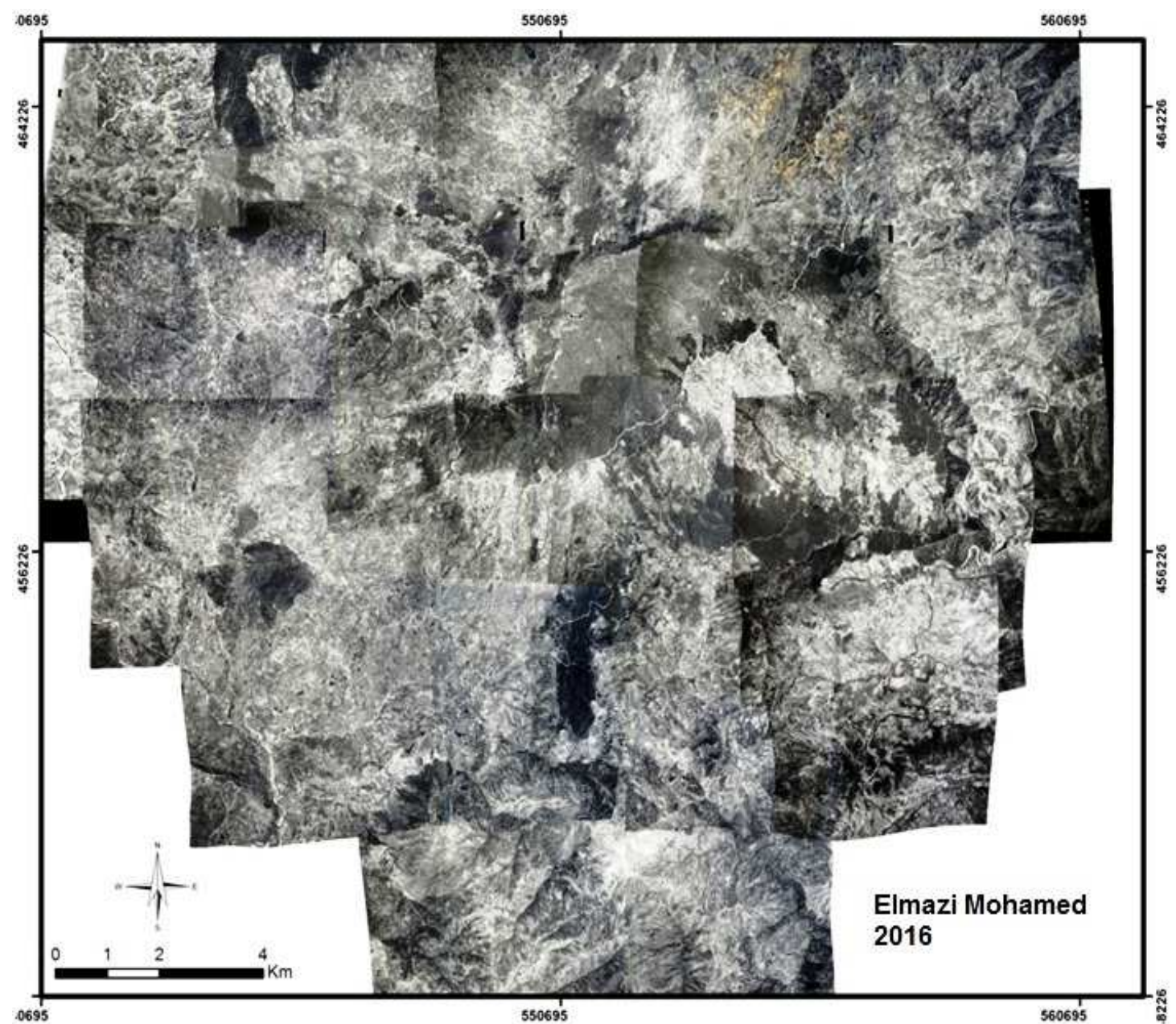

Figure 3 - Mosaïquages des photos aériennes de la zone d'étude

\subsubsection{Photo-interprétation des photographies aériennes de 1962 et 2006 et l'image}

\section{Landsant 2012}

Cette phase compte sur la délimitation des entités homogènes sur les documents du point de vue du recouvrement arboré. Le pourcentage de recouvrement des unités ainsi délimitées est ensuite estimé visuellement en utilisant une charte (Godron et al., 1983). Les caractères du tapis végétal sur les photographies aériennes traduisent assez largement certains aspects écologiques globaux. En effet, les forêts apparaissent sur les photographies aériennes en couleurs foncées et les nuances de cette couleur du foncée au clair indiquent les changements au niveau de l'âge des espèces, du type, de la densité, etc. La haute résolution spatiale de mosaïque facilite l'interprétation grâce à la vue stéréoscopique, en se 
basant sur les critères d'interprétation des photographies aériennes à savoir la teinte, la forme, texture, la densité, la structure, etc. Les résultats obtenus doivent être validés et comparés par le travail du terrain.

\section{Résultats et discussion}

\subsection{Dynamique forestière entre 1962 et 2012}

La méthodologie adoptée dans cette étude, permet de suivre l'évolution récente des peuplements forestiers au niveau du Jbel Outka sur le plan spatial et temporel, à travers la superposition des cartes permettant d'évaluer la dynamique des milieux forestiers durant la période 1962- 2012.

Ainsi, le massif recèle un patrimoine forestier important et diversifié ; il s'étend sur une superficie de 11661 ha en 1962, dont les peuplements endémiques (chêne Tauzin, chêne Zéen) occupent une proportion importante (Fig. 4). Cependant cette forêt subit une dégradation poussée durant les dernières décennies (Fig. 5).

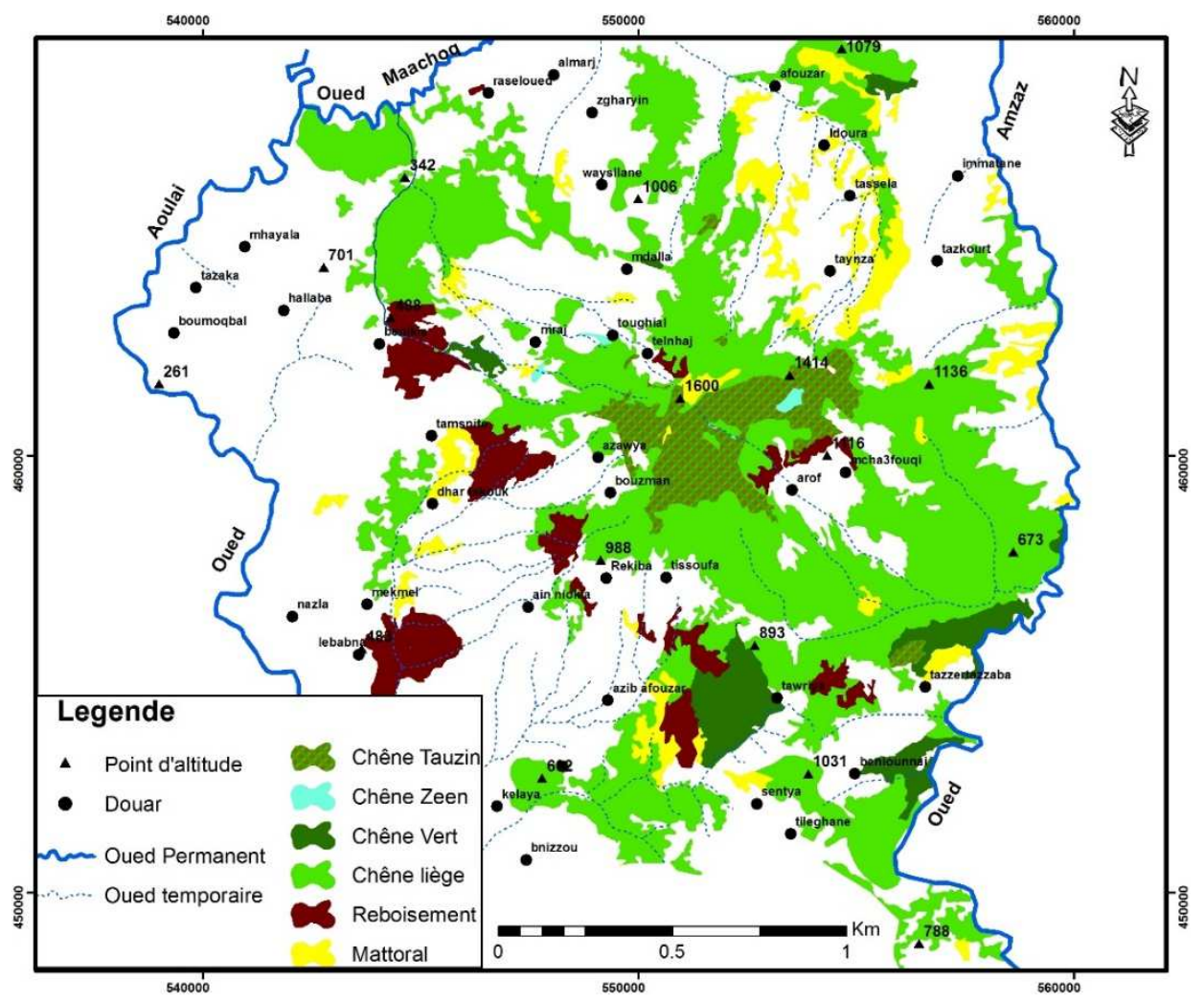

Figure 4 - Recouvrement forestier de Jbel Outka en 1962

Source : Carte réalisée par les auteurs 


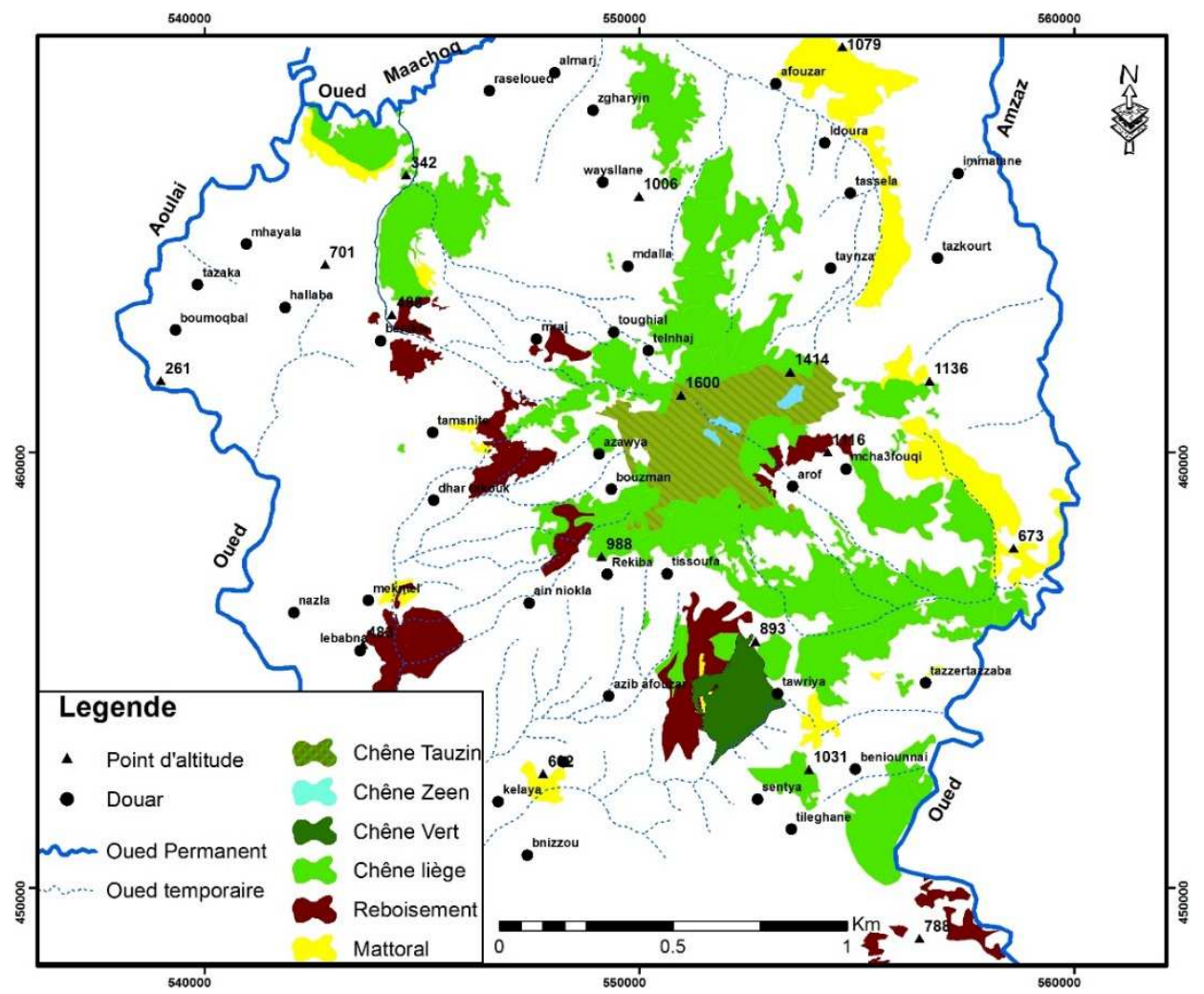

Figure 5 - Recouvrement forestier de Jbel Outka en 2012 Source : Carte réalisée par les auteurs

On constate, à travers les résultats obtenus de l'étude, que la forêt de Jbel Outka a subi une dynamique régressive importante touchant $37,67^{\circ} \%^{\circ}$ de la superficie de cette forêt en 1962. D'une part, les formations naturelles qui se composent essentiellement de Subéraie, de chêne Tauzin et de chêne vert, ont connu une régression très forte atteignant 5179 ha: dont 428 ha transférés au matorral, et 4751 ha défrichés irréversiblement et convertis en terres agricoles, soit un taux de défrichement estimé à 95 ha/an. D'autre part, la superficie de reboisement a progressé de 672 ha en 1962 à 1031 ha en 2006 ; ce qui correspond à un taux de reboisement annuel faible de 9 ha /an seulement (tab 1). 


\begin{tabular}{|c|c|c|c|}
\hline \multirow{2}{*}{ Type de peuplement } & $\begin{array}{c}\text { Superficie } \\
1962(\mathrm{Ha})\end{array}$ & $\begin{array}{c}\text { Superficie } 2016(\mathrm{Ha}) \\
\text { \% évolution } \\
1962-2012\end{array}$ \\
\hline Chêne liège & 7821 & 3433 & $-56,11$ \\
\hline Chêne tauzin & 1262 & 799 & $-36,69$ \\
\hline Chêne Vert & 532 & 213 & $-59,96$ \\
\hline Chêne zéen & 35,01 & 26 & $-25,74$ \\
\hline Reboisement & 672 & 1031 & 53,42 \\
\hline Matorral & 1338 & 1766 & 31,99 \\
\hline Total & 11661 & 7268 & $-37,67$ \\
\hline
\end{tabular}

Tableau 1 - Évolution des peuplements forestiers de la forêt du Jbel Outka entre 1962 et 2012

L'étage du chêne liège est plus étendu dans cette zone et offre les conditions climatiques optimales pour la culture du Kif (Benabid,1997). Durant la période (1962-2012), la forêt de chêne liège a été défrichée sur la quasi-totalité de sa superficie : en 1962, il y en avait 7821 ha ; en 2012, 3433 ha seulement, avec quelques bouquets, par-ci par-là, de chêne liège.

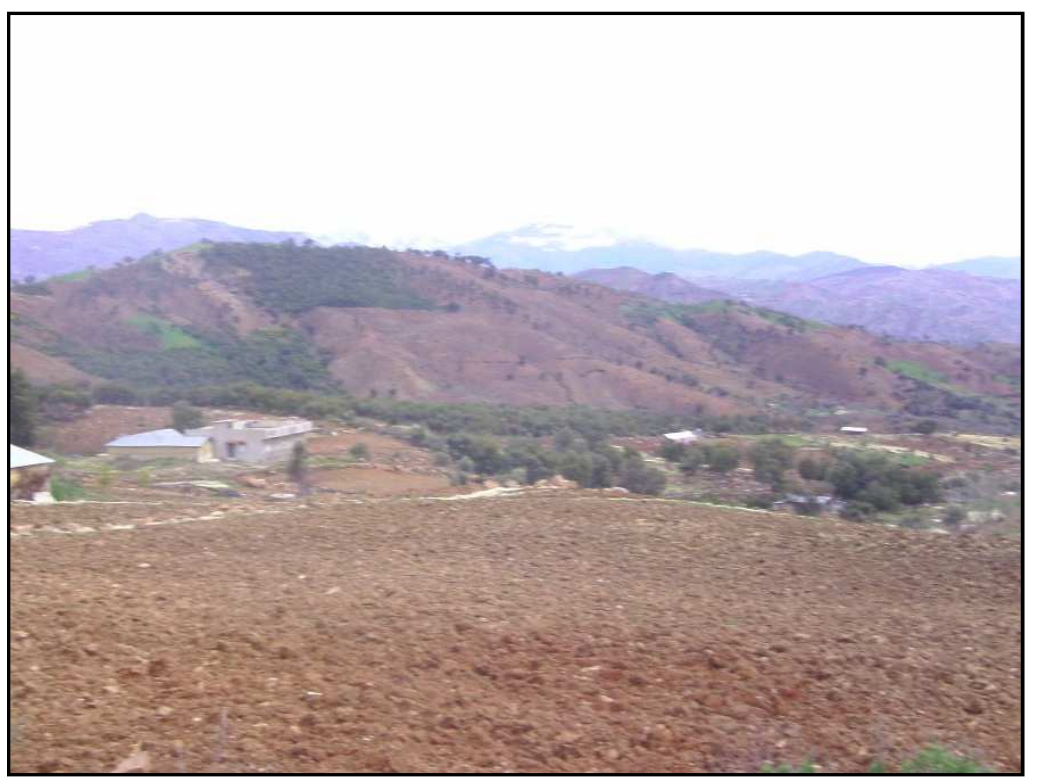

Figure 6 - Un exemple de défrichement de l'étage du chêne liège, converti en terres agricoles (cannabis) sur les versants Sud Est du massif de Jbel Outka.Situation de 2014 
Cette dégradation a touché également les peuplements forestiers qui ont une importance biologique et écologique, notamment le chêne Tauzin qui constitue à Jbel Oudka $34^{\circ}$ de la totalité de Chêne Tauzin au Maroc (Alouane, 1994). Malgré son intérêt il subit une régression alarmante qui affecte plus de $36,69^{\circ}{ }^{\circ}$ de sa superficie par rapport à 1962 . Les $17,05^{\circ}$ ont connu une dynamique semi stable proche du sommet d'Outka grâce à l'existence de la surveillance forestière à l'intérieur du site d'intérêt écologique et biologique Lala Outka. Cependant les espaces replantés ne représentent que $4,57^{\circ}{ }^{\circ}$, et seulement $1,39^{\circ} \%^{\circ}$ a connu une progression dynamique grâce au climat hyper humide.

\subsection{Causes de la dégradation des ressources forestières}

Le domaine forestier de Jbel Outka, est soumis comme partout ailleurs à la fois aux contraintes naturelles et à une forte pression anthropique; celle-ci constitue la cause principale de dégradation des ressources forestières.

\subsubsection{Facteurs naturels}

Les facteurs naturels, à savoir l'érosion du sol et les changements climatiques qui se traduisent par l'augmentation de la température moyenne annuelle, comprise entre $(0,6$ et $1.1^{\circ} \mathrm{C}$ ), et à la récurrence des sécheresses intra-annuelles, annuelles et périodiques, induisent des dysfonctionnements menaçant les espaces forestiers.

En s'appuyant sur l'analyse des données pluviométriques enregistrées durant la période de 1978 à 2013 de la station de Jbel Outka, nous constatons que les précipitations ont enregistré une tendance générale à la baisse, estimée de $150 \mathrm{~mm}$ par rapport à la moyenne annuelle (fig. 7).

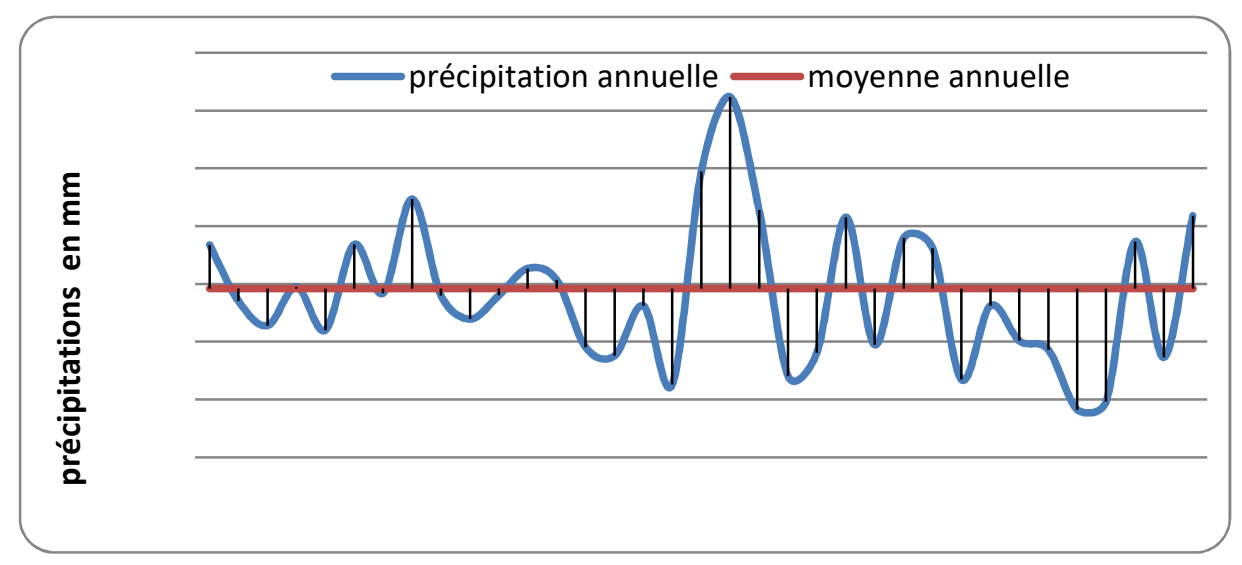

Figure 7 - Précipitations moyennes annuelles enregistrées dans la station de Jbel Outka entre 1978 et 2013

Source : Données de l'Agence du bassin hydraulique du Sebou 
Ces changements observés au niveau de l'augmentation des températures et diminution des précipitations, et la succession des années de sécheresses, parfois sévères, ont eu des effets néfastes sur les écosystèmes forestiers, déjà fragiles, en raison de la pression accrue sur les ressources. Ces impacts sont d'ordre écologique, avec un manque de régénération naturelle conjugué à un vieillissement de ces peuplements, une modification des aires de répartition des espèces et donc des paysages, une forte vulnérabilité aux maladies et aux insectes comme le Bombyx disparate qui, au Maroc, peuple toutes les forêts de Chêne liège (Riyahi. S, in Fraval et Al, 1975). De même, l'augmentation des risques d'incendie constitue les conséquences les plus directes et immédiates du changement climatique sur les forêts dans les montagnes rifains. Ainsi, le taux d'inflammabilité des écosystèmes forestiers est d'autant plus important ; ce qui augmente le déclenchement des feux de forêt.

\subsubsection{Les facteurs anthropiques}

Les études scientifiques effectuées dans les montagnes rifaines montrent que ces pays recèlent un patrimoine forestier important exploité extensivement par les tribus. Néanmoins, depuis l'installation du Protectorat français, la relation de la population avec la forêt a changé. En effet, cette administration forestière a implanté une politique persévérante en vue de constituer un " domaine forestier de l'Etat » par le dahir du 10 octobre 1917 relatif à la conservation et à l'exploitation des forêts, appliquée depuis des années 1926-1930 dans toutes les régions. La réaction des Jbala a été de défricher totalement et durablement de vastes superficies qui étaient livrées jadis à des mises en culture partielles et temporaires (Fay, 1979).

Actuellement, au cours des deux dernières décennies, le domaine forestier dans cette zone montagneuse est soumis à une forte pression anthropique (Fig. 8). L'ampleur de la croissance démographique dans les zones forestières et périforestières a entraîné une forte pression sur l'écosystème forestier. Le recensement de la population et de l'habitat de 2014 indique que la population de la zone d'étude dépasse 8494 habitants. 


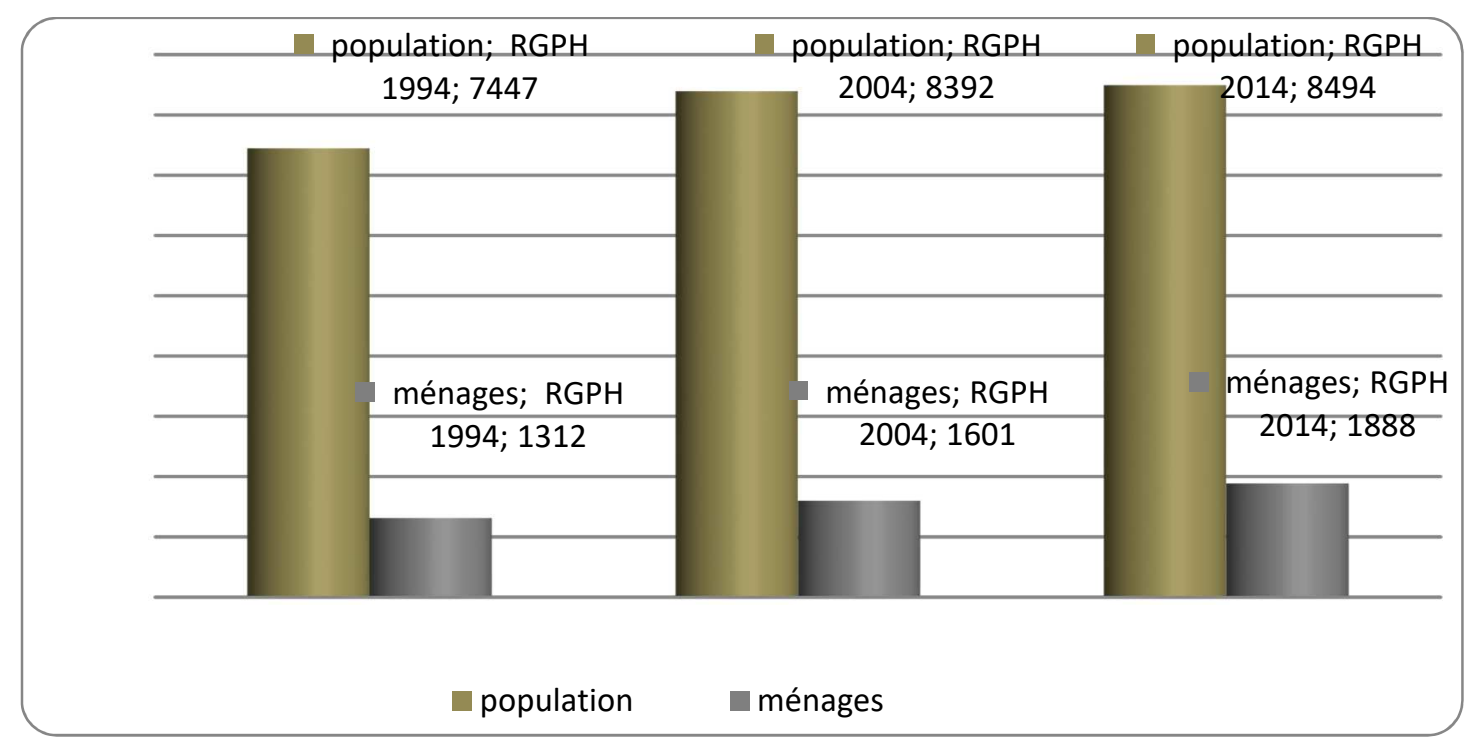

Figure 8 - Evolution de la population de la commune d'Outka entre 1994 et 2014 (RGPH 1994, 2004, 2014)

Les résultats des Recensements généraux de la population et de l'habitat (RGPH) de 1994, 2004 et 2014, indiquent que la population et le nombre de foyers ont connu une évolution importante au cours de ces dernières décennies. Cette population est passée de 7447 habitants en 1994 à 8494 habitants en 2014, soit une évolution de 14,5\% durant cette période. Quant au nombre de ménages, il est passé de 1312 ménages en 1994 à 1888 ménages en 2014, soit une évolution de 29 ménages par an. Cette évolution de la population s'est matérialisée au niveau de la zone d'Outka par la fragmentation des propriétés agricoles et la diminution de la taille des parcelles d'une génération à l'autre par héritage, comme le montre le tableau suivant (Tab. 2).

\begin{tabular}{|c|c|c|c|c|}
\hline \multirow{2}{*}{ Taille en ha } & \multicolumn{2}{|c|}{ Superficie } & \multicolumn{2}{c|}{ Exploitations } \\
\cline { 2 - 5 } & $\mathrm{Ha}$ & $\%$ & Nombre & $\%$ \\
\hline Moins 1 & 663,92 & 8,7 & 1564 & 26,79 \\
\hline $1-2$ & 1686,5 & 22,1 & 1482 & 36,3 \\
\hline $2-3$ & 3258,5 & 42,7 & 1213 & 29,71 \\
\hline $3-4$ & 1533,9 & 20,1 & 265 & 6,49 \\
\hline Sup 4 & 488,4 & 6,4 & 29 & 0,71 \\
\hline Total & 7631 & 100 & 4083 & 100 \\
\hline
\end{tabular}

Tableau 2- La répartition des exploitations selon la taille en ha

(Recensement agricole de 1996) 
On constate de façon générale que la SAU au niveau de la région d'Outka est faible, soit 7631 ha, exploités par 4083 exploitants. Elle se caractérise par la domination des exploitations ayant une taille inférieure à 3 ha, représentant $73,5 \%$ de la SAU, dont les exploitations de moins 2 ha représente une proportion importante d'environ $22.1 \%$; alors que les exploitations ayant plus de 4 ha représentent seulement $6.4 \%$ du total de SAU. En effet, la faible taille des exploitations, implique l'accroissement du besoin de foncier au détriment des forêts par les défrichements, avec l'extension de la culture du cannabis depuis les années 80 du siècle dernier.

Parmi les nombreuses causes de dégradation directe du capital forestier, et en dehors du processus traditionnel d'extension des cultures familiales vivrières, le défrichement pour la culture du kif et la coupe pour le bois de chauffage, représentent près de $90 \%$ du processus de déforestation. L'extension de la culture du cannabis, avec des rendements d'environ 2000 kg/ha (Taiqui, 1997), qui sont "20 à 30 fois supérieurs à ceux d'une bonne récolte d'orge ou le double de la somme moyenne transférée dans l'année par un émigré en Europe" (Maurer, 1992b). Cette extension de la culture de cannabis est responsable de l'avancée spectaculaire du front de déforestation et reste l'ennemi principal du forestier. La rapidité de l'avancée de ce front de déforestation s'explique par le fait que la culture du cannabis est une culture itinérante qui a besoin de bons sols et de beaucoup de soins. Lorsque les sols s'épuisent au bout de 2 à 3 ans environ, l'agriculteur se déplace et défriche de nouvelles terres agricoles au détriment du domaine forestier.

Par ailleurs, les incendies dans le Rif en général et à Jbel Outka en particulier constituent un fléau qui menace la pérennité de la forêt, surtout après l'introduction du Cannabis. En moyenne 16 incendies sont enregistrés annuellement causant la disparition de 58 ha de formation forestière.

D'autres facteurs interviennent dans la dégradation directe du capital forestier mais sont d'ordre secondaire et en régression depuis l'apparition du kif ; ce sont le pâturage en forêt, la coupe de bois d'œuvre et de bois de construction (chêne liège, chêne tauzin, chêne zéen) et l'extraction de produits divers (liège, miel). Ces déforestations découlent des conséquences environnementales, économiques et sociales. 


\section{Conséquences de la dégradation de la couverture forestière}

Les conséquences néfastes et catastrophiques qui découlent de cette forte pression sur les ressources forestières peuvent être résumées comme suit :

- La déforestation dans le Rif accentue l'érosion qui se traduit par la perte d'énormes quantités de matières solides arrachées aux sols et transportées par ruissellement jusqu'au cours d'eau (figure 9), ce qui influe sur les ressources en eau d'une part et sur les retenues des barrages d'autre part, surtout quand on sait que l'érosion spécifique dans le Rif peut atteindre jusqu'au 4000 tonnes $/ \mathrm{km}^{2} /$ an (Laouina, 1994).

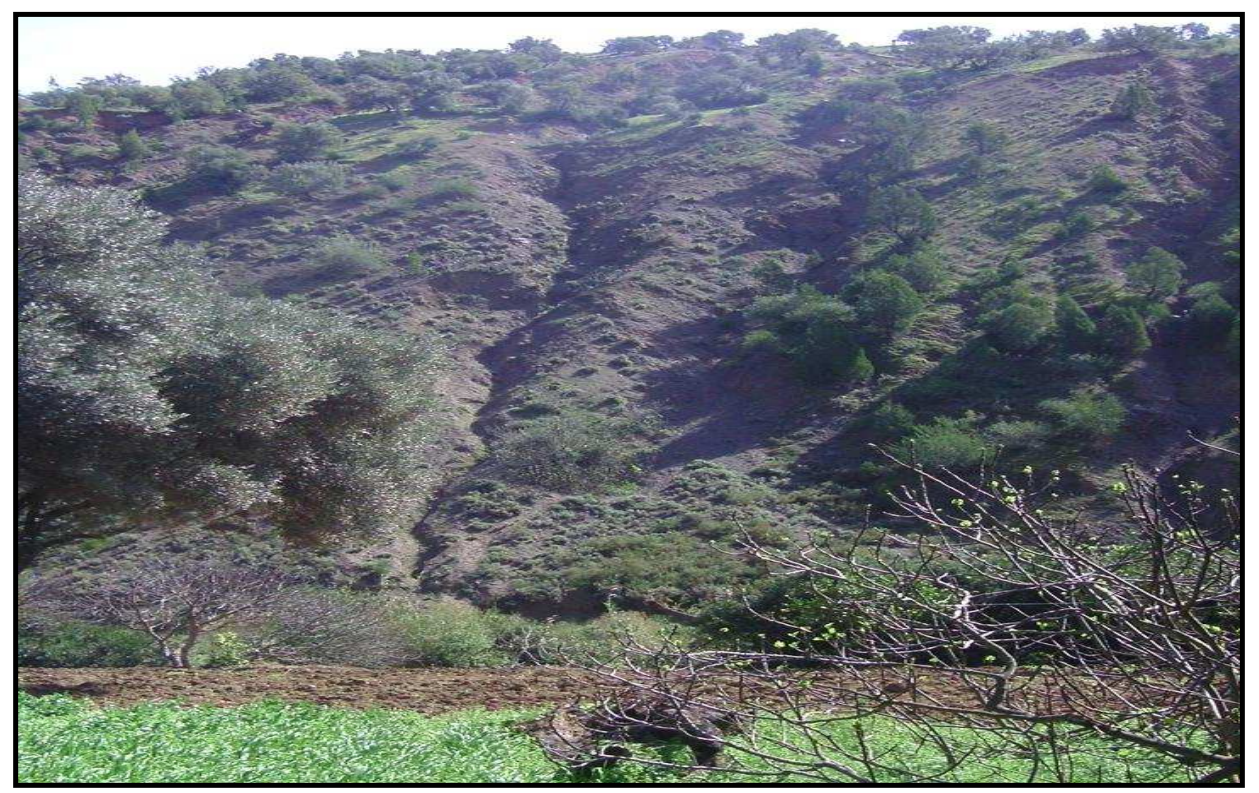

Figure 8 - L'érosion des sols par la destruction de la couverture forestière dans le massif du Jbel Outka. Situation de 2014.

- La dégradation de l’environnement en général et des écosystèmes forestiers en particulier La forêt est un élément central pour optimiser le bilan hydrique du pays. Elle permet de profiter pleinement des pluies abondantes qui s'abattent sur les massifs montagnards (M’hirit et al, 2004). Cependant, le taux de déforestation actuel pourrait avoir rapidement des conséquences irréversibles pour l'approvisionnement en eau dans les décennies à venir.

- Surexploitation des ressources forestières et dégradation de la biodiversité : en effet, les conséquences de la dégradation au niveau de la biodiversité sont innombrables. Depuis le 
début du siècle, la majorité des grandes espèces de différents groupes faunistiques ont disparu ou sont en voie d'extinction (petite Gerbille, porc épais, Loutre, la Genette, Chat Ganté...). Ainsi, les grands écosystèmes forestiers du Rif, comme les oléastraies (Olea europaea, var. silvestris) qui occupaient les sols fertiles à basse altitude sont totalement éteintes et ne sont épargnées qu'au niveau des vestiges maraboutiques (Benabid, 1987). En plus de la déforestation et du surpâturage, les capacités de régénération naturelle de cette formation endémique, notamment le chêne Tauzin et Zéen, sont fortement affaiblies par le desséchement des semis (Taiqui,1997).

- Sur le plan social : la déforestation généralisée réduit l'accès aux produits forestiers nécessaires à la vie de tous les jours, ce qui alourdit encore la tâche des femmes.

\section{Conclusion}

La dégradation observée des écosystèmes forestiers a été efficacement mise en évidence par l'exploitation d'images satellitaires et des photographies aériennes et grâce à I'utilisation des SIG et à la télédétection spatiale. II serait nécessaire de valider cette étude par des mesures plus précises sur le terrain, mais on peut d'ores et déjà dire que les résultats obtenus sont comparés par les observations de terrain.

En se basant sur l'étude de la forêt du Jbel Outka, les résultats de l'étude diachronique montrent que les écosystèmes forestiers dans le Rif central ont subi une régression importante touchant plus de $37,8 \%$ du total; et ce, en comparaison à celui de 1962 . Ainsi tous les peuplements forestiers naturels ont subi un défrichement en relation avec les mutations socio-économiques et sociales surtout après l'extension de la culture du cannabis depuis les années 80 du siècle dernier.

La conservation des écosystèmes forestiers dans les pays rifains exige de nouvelles stratégies de gestion forestière harmonisée et en concertation avec les stratégies socioéconomiques des populations de cette montagne. Parmi les voies à examiner, nous mettons en valeur :, la délimitation du domaine forestier, l'investissement de $20 \%$ des recettes forestières en reboisements par des peuplements productifs (chênes lièges, pins), 
la valorisation des ressources forestières dans le cadre du plan de développement concerté des zones forestières et péri-forestières, l'amélioration des revenus des usagers par l'intensification de l'arboriculture fruitière et de plantes aromatiques et médicinales et par la création d'activités génératrices de revenus (apiculture, safran naturel, etc.), et, enfin, la sensibilisation de la population à la sauvegarde des ressources naturelles, par la mise en place locale d'éducateurs environnementaux

\section{Références bibliographiques}

AALOUANE, Najim. Caractéristiques édaphiques et bioclimatiques du massif forestier du Jbel Oudka (Rif central, Maroc).Th. Doct. Univ, Strasbourg 1,. Strasbourg.1994.

BENABID. Abdelmalek. Etude phytoécologique des peuplements forestiers et préforestiers du Rif centrooccidental (Maroc). Trav. Inst. Se, série Botanique, , 1984, n³4: p. 1-64.

BENABID.A. et. FANANE, Mohamed. Connaissances sur la végétation du Maroc : Phytogéographie, phytosociologie et séries de végétation. Lazaroa 14 , 1994, p. 21-97.

EI MAZI, Mohemed. Dynamique des milieux forestiers et et perspective de protection dans le Rif Central : cas de la forêt de Jbel Outka dans le Rif central : cas de la forêt du Jbel Outka, mémoire du master en Arabe. FES, Département de Géographie, USMBA, 2014.

ER-RIYAHI, Saber. Rhazi, Leila et $A l$,. Utilisation de la télédétection et des systèmes d'information géographique pour l'évaluation de la dynamique du paysage : cas d'une zone boisée de la province de Benslimane (Maroc occidental). Rev. For. Fr. LX, p 657-666.

FAY, Gerard. L'évolution de la paysannerie montagnarde, les Jbala Sud rifains. Méditerranée $n^{\circ} 1-2,1979, \mathrm{p} 81$ 91.

GODRON, M, Daget, P,. LONG, G, et al. Code pour le relevé méthodique de la végétation et du milieu. Paris : CNRS éditions, 1983.

HAMMI, Sanae ., et Al. Évolution des recouvrements forestiers et d'occupation des sols entre 1964 et 2002 dans la haute vallée des Ait Bougumez (Haut Atlas central, Maroc). Revue sécheresse vol 18, 2007, p. 1-7.

LABHAR, Mohamed. Les Espaces forestiers et préforestiers dans le moyen atlas central. revue géographie du Maro, série $n^{\circ} 1-2, N 1-2$, 2002. p 85-96.

LAOUINA, Abdellah. Démographie et dégradation de l'environnement, le cas de la montagne prérifaine. In Rencontre de Tétouan IV. GERM / FLSH Abdelmalek Essaadi. Oct. 1993.

MAURER, G. Montagnes et montagnards au Maghreb (Maroc, Algérie, Tunisie). Evolulion récente du milieu rural. Les Cahiers d'URBAMA, $n^{\circ} 7,1992 b, \mathrm{p}$ 36-61.

MHIRIT., O et BENCHAKROUN, F. Les écosystèmes forestiers et périforestiers : situation, enjeux et perspectives pour 2025. Rabat, 2004.

TAIQUI, Lahcen. La dégradation écologique au Rif marocain : nécessités d'une nouvelle approche, Mediterránea. Serie de estudios biológicos, 2007, p.5-17. 